\title{
Gene expression profiles of Nitrosomonas europaea, an obligate chemolitotroph.
}

\author{
Daniel Arp. \\ Department of Botany and Plant Pathology. \\ Oregon State University, Corvallis OR. 97331 \\ Technical report for product DOE/ER/63149:
}

Nitrosomonas europaea is an aerobic lithoautotrophic bacterium that uses ammonia $\left(\mathrm{NH}_{3}\right)$ as its energy source. As a nitrifier, it is an important participant in the nitrogen cycle, which can also influence the carbon cycle. The focus of this work was to explore the genetic structure and mechanisms underlying the lithoautotrophic growth style of N. europaea.

$N$. europaea converts $\mathrm{NH}_{3}$ to nitrite $\left(\mathrm{NO}_{2}^{-}\right)$by the successive action of ammonia monooxygenase (AMO) and hydroxylamine oxidoreductase (HAO) in a reaction:

$$
\mathrm{NH}_{3}+\mathrm{O}_{2}+2 \mathrm{e}^{-}-\mathrm{AMO} \rightarrow \mathrm{NH}_{2} \mathrm{OH}+\mathrm{H}_{2} \mathrm{O}-\mathrm{HAO} \rightarrow \mathrm{NO}_{2}^{-}+5 \mathrm{H}^{+}+4 \mathrm{e}^{-} \text {; }
$$

of the resulting four electrons, two return to the AMO reaction and two either provide reductant for biosynthesis or pass to a terminal electron acceptor generating energy.

The genome of N. europaea, determined through the DOE Microbial Genome Program, made possible the studies here reported at the levels of whole genome and of single gene categories. The N. europaea genome consists of a single circular chromosome of 2,812,094 base pairs. Genes are distributed evenly around the genome, with $\sim 47 \%$ transcribed from one strand and $\sim 53 \%$ from the complementary strand. A total of 2460 protein-encoding genes emerged from the modeling effort, averaging $1011 \mathrm{bp}$ in length, with intergenic regions averaging $117 \mathrm{bp}$.

Whole genome gene expression. The gene expression profile of cells in exponential growth and during starvation was analyzed using microarrays. During growth, $98 \%$ of the genes increased in expression at least two fold compared to starvation conditions. In growing cells, approximately $30 \%$ of the genes were expressed eight fold higher including genes encoding cytochrome $\mathrm{c}$ oxidase subunit I, cytochrome c, HAO, fatty acid desaturase and other energy harvesting genes. Approximately $10 \%$ were expressed more than 15 fold higher. Approximately 3\% ( 91 genes) were expressed to more than 20 fold of their levels in starved cells including the gene encoding multicopper oxidase type 1. Interestingly, the expression of the genes for AMO increased approximately two fold during growth. During starvation, the bulk of the genes were down-regulated with approximately $60 \%$ conserving low levels of expression (mRNAs) compared to cells in exponential growth. Fewer than 2\% of the genes were expressed more than two fold higher in starved cells. Genes expressed during starvation include those encoding NUDIX hydrolase, tyrosinase, multicopper oxidase, lipoxygenase, cycloxygenase-2, a putative transmembrane protein and other oxidative stress genes (reported at DOE Genomics: GTL. Contractor-Grantee Workshop II. Washington, D.C. February 29 - March 2, 2004.).

Carbon fixation gene expression. N. europaea fixes carbon via the Calvin-Benson-Bassham (CBB) cycle via a type I ribulose bisphosphate carboxylase/oxygenase (RubisCO). The RubisCO operon is composed of five genes, cbbLSQON. This gene organization is similar to the operon for "green-like" type I RubisCOs in other organisms. The $c b b R$ gene coding for the putative regulatory protein for RubisCO transcription was identified upstream of $c b b L$. This study showed that transcription of $c b b$ genes was upregulated when the carbon source was limited, while amo, hao and other energy harvesting related genes were down-regulated. N. europaea responds to carbon limitation by prioritizing resources towards key components for carbon assimilation. Unlike the amo genes, $\mathrm{NH}_{3}$ was not required for the transcription of the $c b b$ genes. All five $c b b$ genes were only transcribed when an external energy source was provided. In actively growing cells, $\mathrm{mRNAs}$ from the five genes in the RubisCO operon were present at different 
levels, probably due to premature termination of transcription, rapid mRNA processing and mRNA degradation (results reported in Wei et al. 2004, Microbiology 150:1869-1879).

Iron related gene expression. Because $N$. europaea has a relatively high content of hemes, sufficient Fe must be available in the medium for it to grow. The genome revealed that approximately $5 \%$ of the coding genes in $N$. europaea are dedicated to Fe transport and assimilation. Nonetheless, with the exception of citrate biosynthesis genes, $N$. europaea lacks genes for siderophore production. The Fe requirements for growth and the expression of the putative membrane siderophore receptors were determined.

$N$. europaea changes its heme content when $\mathrm{Fe}$ is at a relatively low concentration. Biochemical analyses showed that cytochrome and heme contents of cells grown in Fe-limited medium were 4 fold lower than those from Fe-rich medium. Cellular Fe contents (in both membrane and soluble fractions) showed the same trend. The activity of hydroxylamine oxidoreductase was over three fold lower in cells grown in Fe-limited medium than that in full medium. The growth yields at $0.1 \mu \mathrm{M} \mathrm{Fe}$ and at $0.2 \mu \mathrm{M} \mathrm{Fe}$ were about $35 \%$ and $65 \%$ respectively of that observed at $10 \mu \mathrm{M} \mathrm{Fe} \mathrm{(full} \mathrm{Fe} \mathrm{medium).} \mathrm{N.} \mathrm{europaea} \mathrm{has}$ the mechanisms to cope and grow under Fe limitation.

The $N$. europaea genome has 26 sets of genes that are organized similarly to the genes in a fecI/fecR system. Through similarity searches, we have identified possible TonB-dependent Fe receptor genes upor downstream of these sets. Some of these are similar to genes encoding the siderophore receptors for desferrioxamine (desferal), ferrichrome, and coprogen (TABLE 1). In addition to those listed in Table 1, another $20 \mathrm{Fe}$ /siderophore outer membrane receptor genes are identified that are not immediately adjacent to $\sigma$-factor/sensor genes. $N$. europaea also has genes for Fe storage, for Fe ABC transporter, and for other components necessary for Fe uptake.

The addition of desferal (a siderophore commonly produced by soil bacteria) to Fe-limiting medium promoted the growth of $N$. europaea, though with a longer lag phase, suggesting a necessary induction period of the corresponding receptor. A gene for the putative desferal outer membrane receptor was identified by similarity searches (NE1097, a foxA homologue). NE1097 was expressed at a higher level ( $>10$ fold) in Fe-limiting, desferal-containing cultures than in Fe-sufficient cultures. The expression of NE1097 required the presence of desferal, since typical lag phases were observed when inoculants from desferal cultures were used. Several membrane proteins expressed at much higher levels in the cells grown in Fe-limited medium may be involved in Fe transport. For example, a membrane peptide with the calculated MW of the putative desferal receptor was observed only in the cells grown in desferalcontaining medium. Ferric citrate had an effect similar to that of desferal on $N$. europaea growth in Felimiting medium, i.e. with a longer lag phase and a higher final cell density than that in the full medium. Ferrichrome, on the other hand, did not prolong the lag phase, yet increased total cell growth, suggesting that the genes for the ferrichrome receptors were expressed constitutively.

Consistent with the genome sequence data, no siderophores were detected in $N$. europaea culture filtrates under either Fe-limiting or Fe-sufficient conditions using a standard siderophore assay. We considered the possibility that citrate serves as a Fe chelator/siderophore, since $N$. europaea has the necessary genes to produce it. Citrate was detected ( 2 to $5 \mu \mathrm{M})$ in cell-free filtrates from both, low- and full Fe cultures. Surprisingly, cell-free filtrates from full Fe cultures had relatively higher concentrations $(5 \mu \mathrm{M})$ of citrate than in low Fe cultures ( 2 to $3 \mu \mathrm{M})$. The role of citrate in Fe acquisition, if any, is yet to be determined. $N$. europaea apparently expresses siderophore receptors (e.g. NE0731, NE1097) under low Fe conditions to scavenge Fe more efficiently. These results reinforce the notion that $N$. europaea uses siderophores produced by other organisms in natural habitats.

Membrane fractions of cells grown in low and full Fe media were isolated for comparison of the protein composition. Several proteins were expressed much higher in cells grown in Fe-limited media. Analysis of the proteins highly expressed under Fe limiting condition by LC/MS/MS showed that a 
number of TonB-dependent Fe-siderophore outer membrane receptors were the proteins in the predominant bands (Table 2). These proteins include at least six TonB-dependent outer membrane $\mathrm{Fe} /$ siderophore receptors that can be potentially involved in Fe uptake. All these six Fe/siderophore receptor genes are those that are not associated with ECF $\sigma$-factor and sensor genes. As shown in Table 2 , some of them are similar to ferrichrome receptor (NE1089), or catechol/catecholate (enterobactin, NE1540) receptor, but four out of six are unidentified siderophore receptors. This result provides direct evidence for the essential role of the genes and their protein products in Fe acquisition and hence the growth and survival of $N$. europaea under Fe-limiting conditions. Interestingly, an outer membrane protein OmpC (a general diffusion Gram-negative porin) and a type II secretion pathway protein were also identified in the protein bands. These proteins have molecular weights around 78 and $82 \mathrm{kDa}$. $N$. europaea can adapt to low Fe growth environment. Higher expression of these Fe acquisition related proteins in cells grown in low Fe medium provides further biochemical evidence for the adaptation of N. europaea to Fe limiting conditions.

Genes encoding the putative outer membrane desferal receptor (NE1097 and NE1088, foxA homologues) have been cloned, insertional mutant constructs made, and mutant strains obtained through homologous recombination. We have already confirmed that desferal receptor gene is essential for the bacterium to utilize Fe-loaded desferal. Further physiological and genetic characterization of these mutants is in progress (unpublished and reported at DOE Genomics: GTL. Contractor-Grantee Workshop III. Washington, D.C. February 6-9, 2005). 
Table 1. Inventory of genes coding for outer membrane $\sigma$-factor-sensor-receptor systems involved in iron acquisition. (Data from paper under review; Wei et al 2005, J. Bacteriol.)

\begin{tabular}{|c|c|c|c|}
\hline$\sigma$-factor & Sensor & Receptor (homologue)* & Putative siderophore** \\
\hline NE0128 & NE0127 & NE0124/0125/0126 (fecA) & [ferric dicitrate] \\
\hline NE0533 & NE0534 & NE0535 (fhuA) & Ferrichrome \\
\hline NE0541 & NE0542 & NE0546 (fhuA) & Ferrichrome \\
\hline NE0547 & NE0548 & NE0549 (fhuA) & Ferrichrome \\
\hline NE0554 & NE0555 & NE0556 (fhuA) & Ferrichrome \\
\hline NE0557 & NE0558 & NE0559 (fhuA) & Ferrichrome \\
\hline NE0818 & NE0817 & NE0816 & $\mathrm{U}$ \\
\hline NE0980 & NE0979 & NE0978 & Ferrichrome \\
\hline NE1041 & NE1039/1040 & NE1038/1037 & {$[\mathrm{u}]$} \\
\hline NE1071 & NE1070 & NE1063/1062 & {$[\mathrm{u}]$} \\
\hline NE1079 & NE1078 & NE1072 & $\mathrm{U}$ \\
\hline NE1086 & NE1085 & NE1087 (fhuE, fpvA) & ferric coprogen, pyoverdin \\
\hline NE1096 & NE1095 & NE1094/1092 (fhuE) & [hydroxamate] \\
\hline NE1099 & NE1098 & NE1097 (foxA) & Ferrioxamine \\
\hline NE1101 & NE1102 & NE1105/1108 & {$[\mathrm{u}]$} \\
\hline NE1192 & NE1191 & NE1190 & ferric alcaligin, ferripyoverdin \\
\hline NE1217 & NE1218 & NE1220 & {$[\mathrm{u}]$} \\
\hline NE1617 & NE1618/1619 & NE1620/1621 & {$[\mathrm{u}]$} \\
\hline NE1992 & NE1989 & NE1988/1087 & {$[\mathrm{u}]$} \\
\hline NE2138 & NE2137 & NE2140 & $\mathrm{U}$ \\
\hline NE2435 & NE2434 & NE2433 (fhuA) & Ferrichrome \\
\hline NE2486 & NE2485 & NE2484/2482 (fhuE) & [hydroxamate] \\
\hline
\end{tabular}

${ }^{*} f e c A$ homologues are genes for putative iron siderophore outer membrane receptors, many of them are adjacent to fecIR genes.

** The far right column gives putative siderophore types. $\mathbf{u}$, unidentified outer membrane ferric siderophore receptor, and square parentheses indicate that the ORF is probably truncated, interrupted by an IS element, or it is a pseudogene due to a reading frame shift. 
Table 2. Major proteins identified by tandem mass spectrometry that are highly expressed in cells grown in low Fe media. For mass spectrometry peptide identification, selected bands from PAGE were excised and trypsin digested. The digested peptides were run on a Waters Q-TOF (Time of Flight) Ultima Global (Milford, MA). Mascot software (Matrix Science, London, UK) was used to assign the identities (data from paper under review; Wei et al 2005, J. Bacteriol.).

\begin{tabular}{cl}
\hline Gene & \multicolumn{1}{c}{ Protein product and putative function } \\
\hline NE0731* & Fiu, outer membrane receptor for monomeric catechols \\
NE2124* & Fiu, outer membrane receptor for monomeric catechols \\
NE1540* & FepA, outer membrane receptor for ferrienterochelin and colicins \\
NE1089* & $\begin{array}{l}\text { FhuA, ferrichrome receptor, also homologous to FhuE, outer membrane } \\
\text { receptor for ferric coprogen and ferric-rhodotorulic acid }\end{array}$ \\
NE1531* & CirA, outer membrane receptor proteins mostly for Fe transport \\
NE1205* & CirA, outer membrane receptor proteins mostly for Fe transport \\
NE2563 & OmpC, outer membrane protein, general diffusion Gram-negative porins \\
NE1604 & General (type II) secretion pathway (GSP) D \\
NE1548 & CaiA, Acyl-CoA dehydrogenases \\
NE2206 & $\begin{array}{l}\text { ppiD, PpiC-type peptidyl-prolyl cis-trans isomerase, also annotated as SurA, } \\
\text { parvulin-like peptidyl-prolyl isomerase }\end{array}$
\end{tabular}

* These are OM Fe/siderophore receptor genes that are not adjacent to sensor/sigma factor genes. 